\title{
Extended Field Theory. New axioms, Laws and consequences.
}

\author{
Dr. Valentina Markova \\ Institute for Basic Researches, (IBR), Sofia, Bulgaria.
}

\begin{abstract}
The present study attempts to expand the Theory of the Electromagnetic Field to a more general Theory of the Field that also includes the gravitational field. It is known that Maxwell's laws (1864) are based on a single axiom which states that the even movement of a vector E leads to movement in a closed loop (div rot $E=0)$. The author replaces this axiom with a new one, according to which the uneven movement of a vector $E$ leads to an open loop (div rot $E \neq 0$ ) or an open uneven vortex (div Vor $E \neq 0$ ). The derived 2 axioms and 10 laws lead to the following results: even movement is replaced with uneven movement (decelerating or accelerating); movement in a closed loop is replaced with movement in an open loop; movement in $2 D$ leads to movement in 3D; during their movement decelerating vortices emit elementary vortices, while accelerating vortices suck in elementary vortices; a cross vortex generates a longitudinal vortex through special transformation and vice versa- a longitudinal vortex through another special transformation generates a cross vortex.
\end{abstract}

Keywords: Gravitational field, Even rotor, Maxwell's laws, Theory of the Electromagnetic Field, Uneven vortex.

\section{Introduction}

The classic axiom in the Theory of the Electromagnetic Field verifies Maxwell's laws (1864). It postulates that the movement of an electric vector $\mathrm{E}$ in a closed loop is even:

$$
\operatorname{div}(\operatorname{rot} \mathrm{E})=\mathbf{0}
$$

where (rot E) is the movement of the vector $\mathrm{E}$ in a closed loop, div (rot E) is the divergence of the vector $\mathrm{E}$ during its movement in a closed loop (rot E) and the movement of the vector $\mathrm{E}$ in a closed loop (rot E) with zero divergence of the vector $\mathrm{E}$ is equivalent to even movement [1].

The disadvantage of the classic axiom (1) is that it is limited to the description of movements in a closed loop or even movements. For this reason Maxwell's law of electromagnetic induction is presented as follows:

$$
\operatorname{rot} \mathrm{E}=-\mu \partial \mathbf{H} / \partial \mathrm{t}, \text { or } \operatorname{rot} \mathbf{E} \sim \mathbf{H},
$$

where (rot $\mathrm{E}$ ) is the even movement of the electric vector $\mathrm{E}$ in a closed loop, $\mu$ is the coefficient of magnetic permeability, $\partial \mathrm{H} / \partial \mathrm{t}$ is the variation of the magnetic vector $\mathrm{H}$ in time $\mathrm{t}$, and $(\sim)$ is the proportionality between the electric $(\mathrm{E})$ and the magnetic $(\mathrm{H})$ vector [1].

The motivation for altering the classic axiom (1)follows after the need to describe the causal links in uneven movements in open systems, i.e. the necessity to expand the existing laws of the Classic Field Theory[2]. This can be achieved by enriching the knowledge on the classic electromagnetic field and describing a new, open and uneven field with far more diverse and complex dynamics that also includes the gravitational field [3].

\section{Essence. New Axioms}

Definition: The movement of vector (E) in an open loop in a (2D) plain is the movement of vector (E) in (2D) in a closed loop (rot $\mathrm{E})$ where the divergence $\operatorname{div}(\operatorname{rot} \mathrm{E})$ of vector $\mathrm{E}$ is different than zero:

$$
\operatorname{div}(\operatorname{rot} \mathbf{E}) \neq 0 \text {. }
$$

In order to expand the concepts, the notion (1) of movement of vector $\mathrm{E}$ in a closed loop $(\operatorname{div}(\operatorname{rot} E)=0)$ in $2 \mathrm{D}$ (Figure 1a) is replaced by the notion (3) of movement in an open loop (div (rot E) $\neq 0$ in 2D (Figure 1b).

Axiom 1 :The movement of vector (E) in an open loop in $2 \mathrm{D}$ is always uneven and the sequence $\left\{\operatorname{div}\left(\right.\right.$ rot $\left.\left.E_{n}\right)\right\}$ of $n$ discrete values of the vector $E_{n}$ is convergent at $n \rightarrow \infty$.

Therefore Axiom 1 postulates that the movement of vector $\mathrm{E}$ in an open loop is always uneven(Figure 1b).The classic axiom (1) uses the definition of a closed loop (div (rot E) $=0)$ [1]. The new axiom (4) uses a new definition (3) with an open loop (div (rot E) $\neq 0$ [ 2, стр 233- 241 ], [3].

The term vortex (vor) is used in fluid dynamics and defined as "an area in fluids, where the flow rotates evenly along a spiral around an axis line, which can be straight or curved" [4]. To begin with, we can use this classic definition, having in mind that here the vortex (vor) is even. For the purposes of the present study the term must be modified for an uneven vortex or a vortex with uneven movement. Thus the designation of an even vortex "vortex, vor" is replaced with a designation for an uneven vortex "Vortex, Vor" with a capital letter.

Definition: An uneven vortex of the vector $E_{n}\left(\right.$ Vor $\left.E_{n}\right)$ is an even vortex (vor $\left.E_{n}\right)$ for which the sequence $\{$ div $\left(\right.$ Vor $\left.\left.E_{n}\right)\right\}$ of $n$ discrete values of the vector $E_{n}$ is convergent at $n \rightarrow \infty$ or there exists a: 
$\lim \left\{\operatorname{div}\left(\right.\right.$ Vor $\left.\left.\mathrm{E}_{\mathrm{n}}\right)\right\}$ at $\mathrm{n} \rightarrow \infty$.

Definition: An uneven cross vortex $\left(\mathrm{E}_{2 \mathrm{D}}\right)$ is an uneven vortex $(\mathrm{E})$ spinning transversally in a 2D plain. The cross open vortex in 2D is designated as Vortex $E_{2 D}$ or simply Vor $E_{2 D}$ (Figure 1b).

Definition: An uneven longitudinal vortex $\left(\mathrm{H}_{3 \mathrm{D}}\right)$ is an uneven vortex $(\mathrm{H})$ spinning in the volume of 3D.

The longitudinal open vortex in $3 \mathrm{D}$ is designated as Vortex $\mathrm{H}_{3 \mathrm{D}}$ or simply Vor $\mathrm{H}_{3 \mathrm{D}}$ (Figure 1d).Both definitions ignore the thickness of the vortex itself, be it cross or longitudinal.

The differences in geometry are formal and reflect the differences between the cross and longitudinal vortex only superficially. Therefore we will use the abovementioned definitions. For now we can only acknowledge the fact that the longitudinal vortex $\left(\mathrm{H}_{3 \mathrm{D}}\right)$ stands higher in the hierarchy compared to the cross vortex $\left(\mathrm{E}_{2 \mathrm{D}}\right)$. For example the longitudinal vortex $\left(\mathrm{H}_{3 \mathrm{D}}\right)$ is a higher derivative of the cross vortex $\left(\mathrm{E}_{2 \mathrm{D}}\right)$ (Figure 1d).

Axiom 2: Two uneven cross vortex objects in 2D are connected with a single physical cross link in 2D $\left(\mathrm{E}_{2 \mathrm{D}}\right)$.

Since the longitudinal vortex $\left(\mathrm{H}_{3 \mathrm{D}}\right)$ is qualitatively different and represents a higher level in the hierarchy compared to the cross vortex $\left(\mathrm{E}_{2 \mathrm{D}}\right)$, another (longitudinal) link is defined for it.

Axiom 3: Two uneven longitudinal vortex objects in 3D are connected with a single physical longitudinal link in 3D $\left(\mathrm{H}_{3 \mathrm{D}}\right)$.

These three axioms define a complex of composited open systems that manifest both independently and in interaction. The result is a kaleidoscope of forms with diverse dynamics that surprises with many curious findings and conclusions.

Figure 1.The classical axiom is replaced by a new axiom

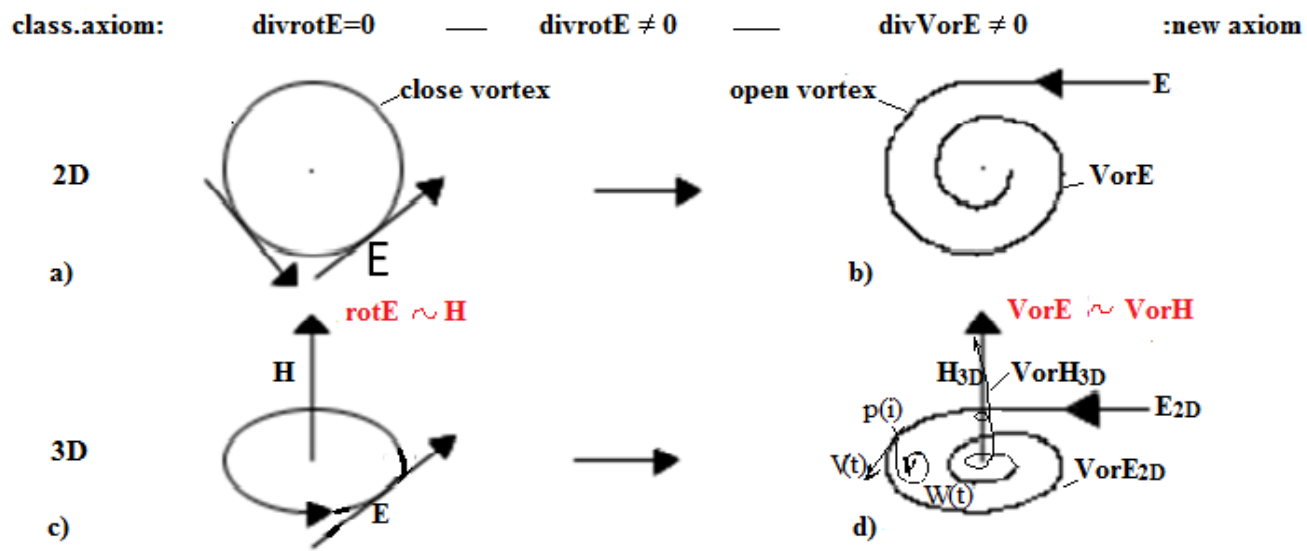

III. Laws Of Transformation (Transformations $\Delta 1, \Delta 2$ )

3.1.Laws of transformation of a velocity vector $(\mathrm{V})$ to vortex amplitude $(\mathrm{W})$ and vice versa.

At every (i) point $\mathrm{p}$ (i) of a decelerating cross vortex $\mathrm{E}$ there are two simultaneous movements: longitudinal (velocity vector $-\mathrm{V}$ ) and transverse (amplitude of the cross vortex-W)(Figure1d,Figure2c). The two simultaneous movements ( $\mathrm{V}$ and $\mathrm{W}$ ) also exist at all points of longitudinal vortices( not Figure).

Law 1: A decelerating vortex $\left(\mathrm{E}_{2 \mathrm{D}^{-}}\right)$with a velocity vector $(\mathrm{V})$ emits to the outside decelerating vortices with amplitude $(\mathrm{W})$ in perpendicular direction.

Figure 2c shows an decelerating cross vortex $\left(\mathrm{E}_{2 \mathrm{D}}{ }^{-}\right)$with a velocity vector $(\mathrm{V})$ that emits to the outside decelerating vortices with amplitude (W) in perpendicular direction.Exactly the opposite dynamics are observed for accelerating (cross or longitudinal) vortices (Figure 2d).

Law 2: An accelerating vortex $\left(\mathrm{E}_{2 \mathrm{D}}+\right)$ with a velocity vector $(\mathrm{V})$ sucks in accelerating vortices with amplitude (W) in perpendicular direction.

At every point $(\mathrm{p})$ of the cross vortex $\mathrm{E}$ there is a velocity vector $(\mathrm{V})$ in $1 \mathrm{D}$ and cross vortex pressure $(\mathrm{W})$ in $2 \mathrm{D}$ (Figure 1d,Figure 2c). Furthermore these movements (V and W) are perpendicular to each other. The peculiarity in this case is that the transformation of the vector $(\mathrm{V})$ into a vortex $(\mathrm{W})$ and vice versa is a nonparametric process. The transformation follows internal laws rather than using parameters set from outside. In every point (p) the nonparametric transformation of two variables $\mathrm{V}(\mathrm{t})$ and $\mathrm{W}(\mathrm{t})$ is mathematically described with the product $\mathrm{V}(\mathrm{t}) \cdot \mathrm{W}(\mathrm{t})$ of these variables( Figure $1 \mathrm{~d})$. 
Law 3: While the velocity vector $(\mathrm{V})$ is transformed into amplitude of the cross vortex $(\mathrm{W})$ ( and vice versa), their complex action at a given time (t) equals the product $\mathrm{V}(\mathrm{t}) \mathrm{W}(\mathrm{t})$ of the velocity vector $\mathrm{V}(\mathrm{t})$ and the amplitude of the cross vortex $\mathrm{W}(\mathrm{t})$ at this moment $(\mathrm{t})$.

In mechanics the simultaneous action of two vectors equals the sum of these vectors, while for the movement of an uneven vortex (point2) the simultaneous action of two vectors $\mathrm{V}(\mathrm{t})$ and $\mathrm{W}(\mathrm{t})$ equals the product of these vectors $\mathrm{V}(\mathrm{t}) \cdot \mathrm{W}(\mathrm{t})$.

3.2.Laws of the transformation of a cross vortex $\left(\mathrm{E}_{2 \mathrm{D}}\right)$ into a longitudinal vortex $\left(\mathrm{H}_{3 \mathrm{D}}\right)$ and vice versa.

The cross vortex $\left(\mathrm{E}_{2 \mathrm{D}^{-}}\right)$is transformed into a longitudinal vortex $\left(\mathrm{H}_{3 \mathrm{D}^{+}}\right)$. This is accomplished through a specific operator $\left(\Delta_{1}\right)$ for cross-longitudinal transformation (Figure 2c).

The cross $\left(\mathrm{E}_{2 \mathrm{D}}\right)$ and the longitudinal $\left(\mathrm{H}_{3 \mathrm{D}}\right)$ vortex are not simply an original entity and an image by analogy with the well-known transformations of Laplace or Fourier. They are representatives of spaces with qualitatively different structures. Therefore the introduced operator $\left(\Delta_{1}\right)$ connects the original in one type (transverse) of space with its image in another type (longitudinal) of space, i.e. the transformation $\Delta_{1}$ connects two spaces with different qualities.

Law 1: The open cross vortex $\left(\mathrm{E}_{2 \mathrm{D}}\right)$ generates an open longitudinal vortex $\left(\mathrm{H}_{3 \mathrm{D}}\right)$ in its center through a crosslongitudinal transformation $\Delta 1$ :

\section{$\Delta 1$}

$\operatorname{Vor}\left(\mathbf{E}_{2 \mathrm{D}}\right)=>--\operatorname{Vor}\left(\mathbf{H}_{3 \mathrm{D}}\right)$,

where Vor (for Vortex, meaning an uneven vortex) replaces rot (for rotor, meaning closed loop) and the cross vortex in $2 \mathrm{D}\left(\mathrm{E}_{2 \mathrm{D}}\right)$ continues its development in $3 \mathrm{D}$ as a longitudinal vortex $\left(\mathrm{H}_{3 \mathrm{D}}\right)$ (Figure 1d,f; Figure 2c).

While Maxwell's law (2) states that vector E generates vector H, the present law (6) postulates that the vortex Vor $\left(\mathrm{E}_{2 \mathrm{D}}\right)$ of $\mathrm{E}$ in $2 \mathrm{D}$ generates a vortex Vor $\left(\mathrm{H}_{3 \mathrm{D}}\right)$ of $\mathrm{H}$ in $3 \mathrm{D}$. The sign (-) for $\operatorname{Vor}\left(\mathrm{H}_{3 \mathrm{D}}\right) 3 \mathrm{D}$ means that $\mathrm{E}_{2 \mathrm{D}}$ and $\mathrm{H}_{3 \mathrm{D}}$ have opposite dynamics.

Definition: An accelerating cross vortex $\left(\mathrm{E}_{2 \mathrm{D}}{ }^{+}\right)$is a cross open vortex $\left(\mathrm{E}_{2 \mathrm{D}}\right)$ for which div (Vor $\left.\mathrm{E}_{2 \mathrm{D}}\right)>0$ where the sequence $\left\{\operatorname{div}\left(\right.\right.$ Vor $\left.\left.\mathrm{E}_{\mathrm{n}}\right)\right\}$ is convergent at $\mathrm{n} \rightarrow \infty$.

Figure $2 b$ shows an accelerating cross vortex $\left(\mathrm{E}_{2 \mathrm{D}}{ }^{+}\right)$.

Definition: An accelerating longitudinal vortex $\left(\mathrm{H}_{3 \mathrm{D}}{ }^{+}\right)$is a longitudinal open vortex $\left(\mathrm{H}_{3 \mathrm{D}}\right)$ for which div (Vor $\left.\mathrm{H}_{3 \mathrm{D}}\right)>0$ where the sequence $\left\{\operatorname{div}\left(\right.\right.$ Vor $\left.\left.\mathrm{E}_{\mathrm{n}}\right)\right\}$ is convergent at $\mathrm{n} \rightarrow \infty$.

Figure $2 \mathrm{c}$ shows an accelerating longitudinal vortex $\left(\mathrm{H}_{3 \mathrm{D}}{ }^{+}\right)$.

Definition: A decelerating cross vortex $\left(\mathrm{E}_{2 \mathrm{D}}{ }^{-}\right)$is a cross open vortex $\left(\mathrm{E}_{2 \mathrm{D}}\right)$ for which $\operatorname{div}\left(\operatorname{Vor} \mathrm{E}_{2 \mathrm{D}}\right)<0$ where the sequence $\left\{\operatorname{div}\left(\right.\right.$ Vor $\left.\left.\mathrm{E}_{\mathrm{n}}\right)\right\}$ is convergent at $\mathrm{n} \rightarrow \infty$.

Figure 2a shows a decelerating cross vortex $\left(\mathrm{E}_{2 \mathrm{D}}{ }^{-}\right)$.

Definition: A decelerating longitudinal vortex $\left(\mathrm{H}_{3 \mathrm{D}}{ }^{-}\right)$is a longitudinal open vortex $\left(\mathrm{H}_{3 \mathrm{D}}\right)$ for which div $\left(\mathrm{Vor}_{3 \mathrm{D}}\right)$ $<0$ where the sequence $\left\{\operatorname{div}\left(\operatorname{Vor} \mathrm{E}_{\mathrm{n}}\right)\right\}$ is convergent at $\mathrm{n} \rightarrow \infty$.

Figure $2 \mathrm{~d}$ shows a decelerating longitudinal vortex $\left(\mathrm{H}_{3 \mathrm{D}}{ }^{-}\right)$.

The present paper describes only the process of generation of matter i.e. only the decelerating and consuming half (Figure 2c) of the generator-

consumer chain

(Figure 2c;Figure 2d). The decelerating cross vortex $\left(\mathrm{E}_{2 \mathrm{D}}{ }^{-}\right)$generates an accelerating longitudinal vortex $\left(\mathrm{H}_{3 \mathrm{D}}{ }^{+}\right)$ in its center through a physical transformation $(\Delta 1-)$ (Figure 2c). This transformation is achieved through a new phenomenon called full resonance (resonance in amplitude, frequency and phase).

Consequence : The open decelerating cross vortex $\left(\mathrm{E}_{2 \mathrm{D}}{ }^{-}\right)$generates an open accelerating longitudinal vortex $\left(\mathrm{H}_{3 \mathrm{D}}{ }^{+}\right)$in its center through a cross-longitudinal transformation $\Delta 1$-:

$\Delta 1-$

Figure 2c shows this transformation in $3 \mathrm{D}$.

$\operatorname{Vor}\left(\mathbf{E}_{2 \mathrm{D}}{ }^{-}\right)=>\operatorname{Vor}\left(\mathbf{H}_{3 \mathrm{D}}{ }^{+}\right)$

Law 1(point3.2.) is relevant to the consuming and decelerating part of the cross vortex chain (Figure 2a, c). Law 1 and the Consequence describe entirely and coherently only the Electromagnetic Field.

For the opposite transformation a new operator $\Delta 2$ is introduced to transform a longitudinal $\left(\mathrm{H}_{3 \mathrm{D}}\right)$ into a cross $\left(\mathrm{E}_{2 \mathrm{D}}\right)$ vortex. The physical nature of this $\Delta 2$ transformation is quite different in comparison with $\Delta 1$. Generally speaking, the transformations $\Delta 1$ and $\Delta 2$ are orthogonal rather than symmetrical to each other. 
Figure 2.System of two pair vortices-in 2D and in 3D

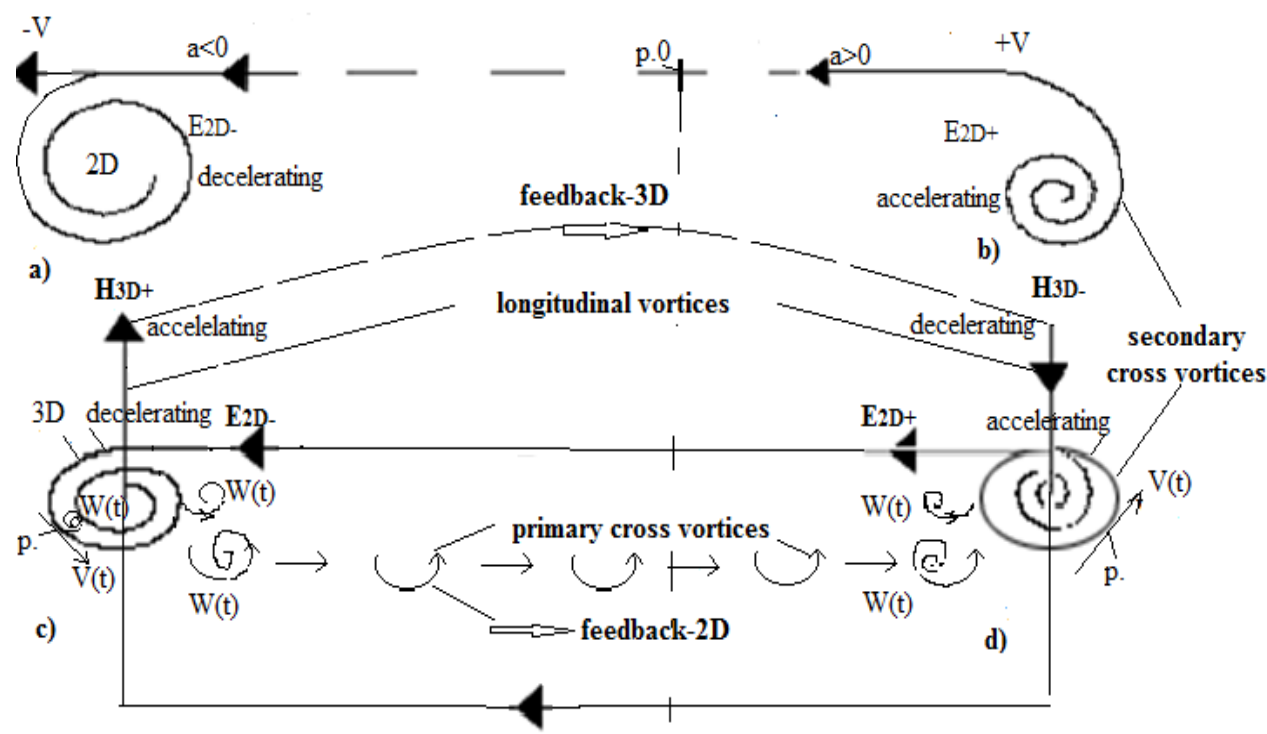

For the opposite transformation of Law 2(point3.2.) a new operator $\Delta 2$ is introduced to transform a longitudinal $\left(\mathrm{H}_{3 \mathrm{D}}\right)$ into a cross $\left(\mathrm{E}_{2 \mathrm{D}}\right)$ vortex. The physical nature of this $\Delta 2$ transformation is quite different in comparison with $\Delta 1$. Generally speaking, the transformations $\Delta 1$ and $\Delta 2$ are orthogonal rather than symmetrical to each other.

Law 2: The open longitudinal vortex $\left(\mathrm{H}_{3 \mathrm{D}}\right)$ generates an open cross vortex $\left(\mathrm{E}_{2 \mathrm{D}}\right)$ in its center through a longitudinal-cross transformation $\Delta 2$ :

\section{$\Delta 2$}

$\operatorname{Vor}\left(\mathbf{H}_{3 \mathrm{D}}\right)=>$-- Vor $\left(\mathbf{E}_{2 \mathrm{D}}\right)$

Law 2 is relevant to the generating part of the transverse chain (Figure 2b, Figure2d).

Consequence: The open decelerating longitudinal vortex $\left(\mathrm{H}_{3 \mathrm{D}}^{-}\right)$generates an open accelerating cross vortex $\left(\mathrm{E}_{2 \mathrm{D}}{ }^{+}\right)$in its center through a longitudinal-cross transformation $\Delta 2-$ :

$\Delta 2$ -

$\operatorname{Vor}\left(\mathbf{H}_{3 \mathrm{D}-}\right)=>\operatorname{Vor}\left(\mathbf{E}_{2 \mathrm{D}+}\right)$

This transformation is presented on Figure $2 \mathrm{~d}$.

Law 2 (point3.2.) is relevant to the generating and accelerating part of the cross vortex chain (Figure 2b,d). Law 2 and the Consequence describe another field, with inverse property as the Electromagnetic Field.

3.3Conclusions

Law 1 (point 3.2.) is relevant for the consuming transformation $\Delta 1$ and, in particular, to the electromagnetic field (Figure 2c).

Law 2 (point 3.2) is relevant for the generating transformation $\Delta 2$ when the movement of the longitudinal vortex $\left(\mathrm{H}_{3 \mathrm{D}}\right)$ is the cause (generator) and the movement of the cross vortex $\left(\mathrm{E}_{2 \mathrm{D}}\right)$ is the effect.

Obviously the two transformations $\Delta 1$ (Law1) and $\Delta 2$ (Law2) are not symmetrical but rather form pairs of objects that complement each other in their action i.e. the pairs are orthogonal. Both transformations, $\Delta 1$ (Law1) and $\Delta 2$ (Law2), are nonparametric. Therefore the processes are regulated by internal laws and are not determined by outside parameters.

In the described vortex pairs (Figure 2c; Figure2d) one vortex plays the role of generator (Figure 2d) and the other of consumer (Figure $2 \mathrm{c}$ ). The generator can emit $\left(\mathrm{E}_{2 \mathrm{D}}+\right)$ to the outside (Figure $2 \mathrm{~d}$ ) or suck to the inside (no figure), but is always accelerating. The consumer can suck $\left(\mathrm{E}_{2 \mathrm{D}^{-}}\right)$(Figure $2 \mathrm{c}$ ) in or emit (no figure) but is always decelerating.

\section{Laws Of Continuity}

Axiom 2 and Axiom 3 (point 2) resemble an axiom in Euclidean geometry but in essence they are physical, rather than geometrical. Instead of points as geometric objects there is a pair of vortices with different dynamics as physical objects; one of them is a generator (accelerating vortex) and the other is a consumer (decelerating vortex). 
4.1.Law of continuity in a closed loop in $2 \mathrm{D}$

Provisionally vortices can be classified as primary (W) and secondary (E) uneven vortices. The primary uneven vortices are microvortices (W) and the secondary uneven vortices are macrovortices (E) (Figures 1d; (Figures $2 \mathrm{c}, \mathrm{d})$

Definition: Primary vortices (W) are emitted to the outside or sucked in by a main vortex (E), also called a secondary vortex.

For example a decelerating cross vortex $\left(\mathrm{E}_{2 \mathrm{D}}{ }^{-}\right)$emits decelerating primary cross vortices in perpendicular direction (Figure 2c). In general the primary microvortices are derivatives of the main secondary macrovortices. The relationship between primary and secondary vortices resembles the one between the magnetic and the Electric field. The Magnetic field, however, is the first derivative of the Electric field, while primary vortices are higher derivatives (second, third, etc.) of the main secondary vortices.

Since cross vortex objects are physical, they must fulfill the main law of continuity and cycle of movement.

Axiom 2 (point 2) considers the question of the link in the opposite direction which closes the full circle (loop) of cross vortices in $2 \mathrm{D}$. The only answer is that in order to fulfill the fundamental law of continuity, the feedback must pass through empty space (feedback 2D) containing elementary primary cross vortices generated and emitted by the secondary decelerating cross vortex(Figure 2c) and consumed and sucked in by the secondary accelerating cross vortex (Figure 2d). This feedback is somewhat imaginary and it closes through the cross vortices. Therefore, in order to satisfy this fundamental law in physics, apparently this space cannot be "empty", as we often call it. The imaginary space is filled with primary cross vortices: copies of the secondary cross vortices but at a much smaller scale.

Law: A pair of open vortex objects in 2D (secondary cross vortices) forms a closed loop in 2D. It transfers matter and vortex energy in one real direction through a real physical link: $\mathrm{E}_{2 \mathrm{D}}(+) \div \mathrm{E}_{2 \mathrm{D}}(-)$, (Figure $2 \mathrm{~d} \div$ Figure 2c), and in the opposite direction through imaginary feedback (feedback 2D) in the space between these objects: (Figure $2 \mathrm{c} \div$ Figure $2 \mathrm{~d}$ ) filled with primary cross vortices.

The reason for the emission of primary elementary cross vortices is the deceleration of the main longitudinal vortex $\left(\mathrm{E}_{2 \mathrm{D}}{ }^{-}\right)$(Figure $2 \mathrm{c}$ ), while their movement in the space between the two vortex objects in $2 \mathrm{D}$ is due to the sucking action of the accelerating main longitudinal vortex $\left(\mathrm{E}_{2 \mathrm{D}}{ }^{+}\right)$(the second vortex in the pair) (Figure $2 b)$.

4.2.Law of continuity in a closed loop in 3D

Axiom 3 (point 2.) considers the nature of the link in the opposite direction that closes the full circle (loop) of main longitudinal vortices in 3D perpendicular to the circle (loop) in 2D. The only answer is that in order to fulfill the fundamental law of physics the feedback of the main longitudinal vortices in 3D must close through the space (feedback 3D) containing elementary primary longitudinal vortices, generated and consumed by the main secondary longitudinal vortices. This imaginary space is filled with primary longitudinal vortices resembling copies of the secondary longitudinal vortices but at a much smaller scale. All longitudinal vortices (primary and secondary) create a new type of field that contributes to our knowledge of the field as a form of matter.

Law: A pair of open vortex objects in 3D (secondary longitudinal vortices) forms a closed loop in 3D. It transfers energy in one direction through the main transverse link $\mathrm{H}_{3 \mathrm{D}}(-) \div \mathrm{H}_{3 \mathrm{D}}(+)$ (Figure $2 \mathrm{~d} \div$ Figure $2 \mathrm{c}$ ), and in the opposite direction through open feedback (feedback 3D) in the space filled with primary cross vortices between these objects (Figure 2c $\div$ Figure 2d).

The reason for the emission of primary elementary longitudinal vortices is the break-up by high frequency pulses (Figure 2e) during the accelerating motion of the secondary longitudinal vortex $\left(\mathrm{H}_{3 \mathrm{D}}{ }^{+}\right)$(Figure $2 \mathrm{c})$. The reason for their movement in the space between the pair of vortex objects in $3 \mathrm{D}$ is the sucking action of the decelerating secondary longitudinal vortex $\left(\mathrm{H}_{3 \mathrm{D}}{ }^{-}\right)$(the second vortex in the pair) (Figure 2d).

The fact of radiating primary vortices of a decelerating vortex and sucking in primary vortex by accelerating vortex has probably been known for a long time. Certainly, Nikola Tesla has applied this knowledge to construct apparatus using the energy of these primary vortices.

The real links in 2D and 3D are simple, but the imaginary links in 2D (feedback 2D) and 3D (feedback 3D) are most likely multiciphered.

\subsection{Law of continuity in two mutually perpendicular closed loops in 2D and 3D}

Feedback (2D and 3D) connects a pair of different vortex objects (Figure $2 \mathrm{c}, \mathrm{d}$ ). When these objects include both cross and longitudinal vortices, connected internally with cause-and-effect transformations, either $(\Delta 1)$ or $(\Delta 2)$, they are called complex vortex objects.

Definition: A complex vortex object is an object that contains a cross and a longitudinal vortex connected internally by cause-and-effect transformations, either $(\Delta 1)$ or $(\Delta 2)$. 
Law: Two complex vortex objects are connected with mutually perpendicular closed loops in $2 \mathrm{D}$ and 3D. The closed loop: $\left\{\mathrm{E}_{2 \mathrm{D}}(+) \div \mathrm{E}_{2 \mathrm{D}}(-)\right\} \div\left\{(\right.$ feedback(2D) $\}$ is perpendicular to the closed loop: $\left\{\mathrm{H}_{3 \mathrm{D}}(-) \div \mathrm{H}_{3 \mathrm{D}}(+)\right\}$ $\div\{$ (feedback (3D) $\}$, (Figure 2c,d).

\section{Laws Of Motion Of Longitudinal-Cross Vortices}

An accelerating longitudinal vortex has acceleration A (Figure $3 \mathrm{a}$, b). Let us consider a particular accelerating longitudinal vortex with acceleration $(+\mathrm{A})$ and increasing velocity: $\mathrm{V}_{1}, \mathrm{~V}_{2}, \ldots, \mathrm{V}_{\mathrm{n}}$, increasing acceleration of cross vortices: $\mathrm{e}_{1}, \mathrm{e}_{2}, \ldots \mathrm{e}_{\mathrm{n}}$, and decreasing amplitude of the cross vortices $\mathrm{W}_{1}, \mathrm{~W}_{2}, \ldots \mathrm{W}_{\mathrm{n}}$ (Figure $3 a)$. Let us also consider a particular decelerating longitudinal vortex with acceleration (-A) with decreasing velocity: $\mathrm{V}_{1}, \mathrm{~V}_{2}, \ldots, \mathrm{V}_{\mathrm{n}}$, decreasing acceleration of cross vortices: $\mathrm{e}_{1}, \mathrm{e}_{2}, \ldots \mathrm{e}_{\mathrm{n}}$, and increasing amplitude of the cross vortices $\mathrm{W}_{1}, \mathrm{~W}_{2}, \ldots \mathrm{W}_{\mathrm{n}}$ (Figure $3 \mathrm{~b}$ ). The acceleration and deceleration of the longitudinal vortex is a nonparametric process. Accelerating and decelerating longitudinal vortices do not manifest qualitative differences. They only differ quantitatively by their magnitude and sign of the change [5].

5.1.Law of the velocity of the longitudinal vortex (V) and the amplitude of the cross vortices (W)

Law: The velocity $(\mathrm{V})$ of an accelerating longitudinal vortex increases in (n) portions $(\psi)^{\mathrm{n}}$ times while the amplitude (W) of cross vortices decreases reciprocally in (n) portions $(1 / \psi)^{\mathrm{n}}$ times; the velocity of a decelerating longitudinal vortex decreases in (n) portions $(1 / \psi)^{\mathrm{n}}$ times, while the amplitude (W) of cross vortices increases reciprocally in (n) portions $(\psi)^{\mathrm{n}}$ times:

$$
\mid \begin{array}{ll}
\mid \mathbf{V}^{2}=\mathbf{V}_{0}(1+\mathbf{V}) & \\
\mid \mathbf{W}^{2}=\mathbf{W}_{0}(1-\mathrm{W}), & \mid \begin{array}{l}
\mathbf{V}^{2}=\mathbf{V}_{0}(\mathbf{1}-\mathbf{V}) \\
\mathbf{W}^{2}=\mathbf{W}_{0}(\mathbf{1}+\mathbf{W})
\end{array}
\end{array}
$$

where $\mathrm{v}_{\mathrm{n}}$ and $\omega_{\mathrm{n}}$ are periodic roots with period $\mathrm{n}$ that fulfill the requirement for orthogonality: $\mathrm{v}_{\mathrm{n}} \omega_{\mathrm{n}}=\mathrm{V}_{0} \cdot \mathrm{W}_{0} ; \mathrm{n}=$ $0 \div \infty$; the roots $\mathrm{V}_{\mathrm{n}}$ and $\omega_{\mathrm{n}}$ are expressed as: $\mathrm{v}_{\mathrm{n}}=\psi^{\mathrm{n}} \cdot \mathrm{V}_{0}, \mathrm{w}_{\mathrm{n}}=(1 / \psi)^{\mathrm{n}} \cdot \mathrm{W}_{0} ; \mathrm{V}_{0}$ is the starting value of $\mathrm{V}_{\mathrm{n}}, \mathrm{W}_{0}$ is the starting value of $\mathrm{W}_{\mathrm{n}}$ and $\psi$ is a number that fulfills the requirement: $\psi-1 / \psi=1$.

The first positive root of the first equation (8a) is $\mathrm{v}_{1}=\psi \cdot \mathrm{V}_{0}=1,62 . \mathrm{V}_{0}$. The first positive root of the second equation (8b) is: $\mathrm{w}_{1}=1 / \psi \cdot \mathrm{W}_{0}=0,62 \cdot \mathrm{W}_{0}$. The periodic roots of the first equation (8a) are obtained from

the expression $\mathrm{V}^{\mathrm{n}}=\mathrm{V}_{0}\left(\mathrm{~V}^{\mathrm{n}-1}+\mathrm{V}^{\mathrm{n}-2}\right)$. The periodic roots of the second equation (8b) are obtained from the expression $\mathrm{W}^{\mathrm{n}-2}=\mathrm{W}_{0} \cdot\left(\mathrm{W}^{\mathrm{n}}-\mathrm{W}^{\mathrm{n}-1}\right)$.

The present paper considers the simultaneous action of both equations in systems (8),(9). They describe a qualitatively new, specific and combined movement in the form of a system:

The system expresses the simultaneous action of two vortex movements - longitudinal and transverse;

The system is orthogonal, i.e. the direction of the velocity of the longitudinal vortex $\mathrm{V}$ is perpendicular to the direction of the amplitude $\mathrm{W}$ of the cross vortices and to the direction in which the cross vortices are sucked in, respectively;

The system is open (as opposed to closed), i.e. the system has a closed internal part, the longitudinal vortex, and an open external part, the cross vortices.

\subsection{Law of the constant power of the longitudinal vortex}

According to Axiom 2 and Axiom 3 (point 2) at every cycle (i) the accelerating longitudinal vortex $\left(\mathrm{A}_{\mathrm{i}}\right)$ sucks in portions (quanta) of cross vortices $\left(\mathrm{e}_{1}<\mathrm{e}_{2}<\mathrm{e}_{3}<, \ldots\right)$ with amplitude $\left(\mathrm{W}_{\mathrm{i}}\right)$ (Figure 3a), while the decelerating longitudinal vortex $\left(\mathrm{A}_{\mathrm{i}}\right)$ emits portions (quanta) of cross vortices $\left(\mathrm{e}_{1}<\mathrm{e}_{2}<\mathrm{e}_{3}<, \ldots\right)$ with amplitude $\left(\mathrm{W}_{\mathrm{i}}\right)($ Figure 3b) [4].

In the case of the accelerating longitudinal vortex $(\mathrm{A}+)$ the velocity increases $(\mathrm{V}+)$, while the amplitude of the cross vortices decreases (W-) in such a way that their product $(\mathrm{V}+) .(\mathrm{W}-)$ remains constant all along the longitudinal vortex $(\mathrm{A}+)$ (Figure $3 \mathrm{a})$. The product $(\mathrm{V}+) .(\mathrm{W}-)$ is proportional to the power of the accelerating longitudinal vortex $(\mathrm{A}+)$.

In the case of the decelerating longitudinal vortex (A-) the velocity decreases (V-), while the amplitude of the cross vortices increases $(\mathrm{W}+)$ in such a way that their product $(\mathrm{V}-) .(\mathrm{W}+)$ remains constant all along the longitudinal vortex (Figure $3 \mathrm{~b})$. The product $(\mathrm{V}-) .(\mathrm{W}+)$ is proportional to the power of the decelerating longitudinal vortex (A-).

Law: For an uneven (accelerating or decelerating) longitudinal vortex $(A)$ with current velocity $\left(\mathrm{V}_{\mathrm{i}}\right)$ and amplitude of the cross vortices $\left(\mathrm{W}_{\mathrm{i}}\right)$ the product $\left(\mathrm{V}_{\mathrm{i}}\right) .\left(\mathrm{W}_{\mathrm{i}}\right)$ is a constant:

$$
\left(\mathbf{V}_{\mathrm{i}}\right) \cdot\left(\mathbf{W}_{\mathrm{i}}\right)=\text { const., }
$$

where $\mathrm{i}=0 \div \infty$ and the product $\left(\mathrm{V}_{\mathrm{i}}\right) .\left(\mathrm{W}_{\mathrm{i}}\right)$ is proportional to the power of the uneven longitudinal vortex $(\mathrm{A})$. 


\subsection{Consequences}

By analogy with the main Law 1 (point 3.2) of transformation we can write down the following transformation Vor e $\rightarrow \Delta 1 \rightarrow-$ Vor h (Figure 3a). The different signs in this transformation show that (Vor e) and (Vor h) have opposite dynamics. For example if (Vor e) is accelerating, then (Vor h) is decelerating (Figure 3a) and vice versa (Figure 3b). Furthermore (Vor h) is perpendicular to (Vor e) (Figure $3 \mathrm{a}, \mathrm{b}$ ).

Accelerating cross vortices $\left(\mathrm{e}_{\mathrm{i}}\right)$ move from outside in (-Vor e). Therefore the direction of the longitudinal vortex (-Vor h) is also from outside in (due to the right-oriented vector system or the right-hand rule). Because of the directions of (-h) from all vortices (-Vor $h)$, the central accelerating longitudinal vortex (A) is winding clockwise (+) when the observer(obs.) is facing opposite to the direction of the movement (Figure 3a).

Decelerating cross vortices $\left(\mathrm{e}_{\mathrm{i}}\right)$ move from inside out $(+\mathrm{Vor} \mathrm{e})$. Therefore the direction of the longitudinal vortex (-Vor h) is also from inside out (due to the right-oriented vector system or the right-hand rule). Because of the directions of $(+\mathrm{h})$ from all vortices $(+$ Vor $h)$, the central accelerating longitudinal vortex (A) is winding counterclockwise (-) when the observer(obs.) is facing opposite to the direction of the movement (Figure 3b).

Figure 3.A system of bidirectionals accelelating and decelerating vortices

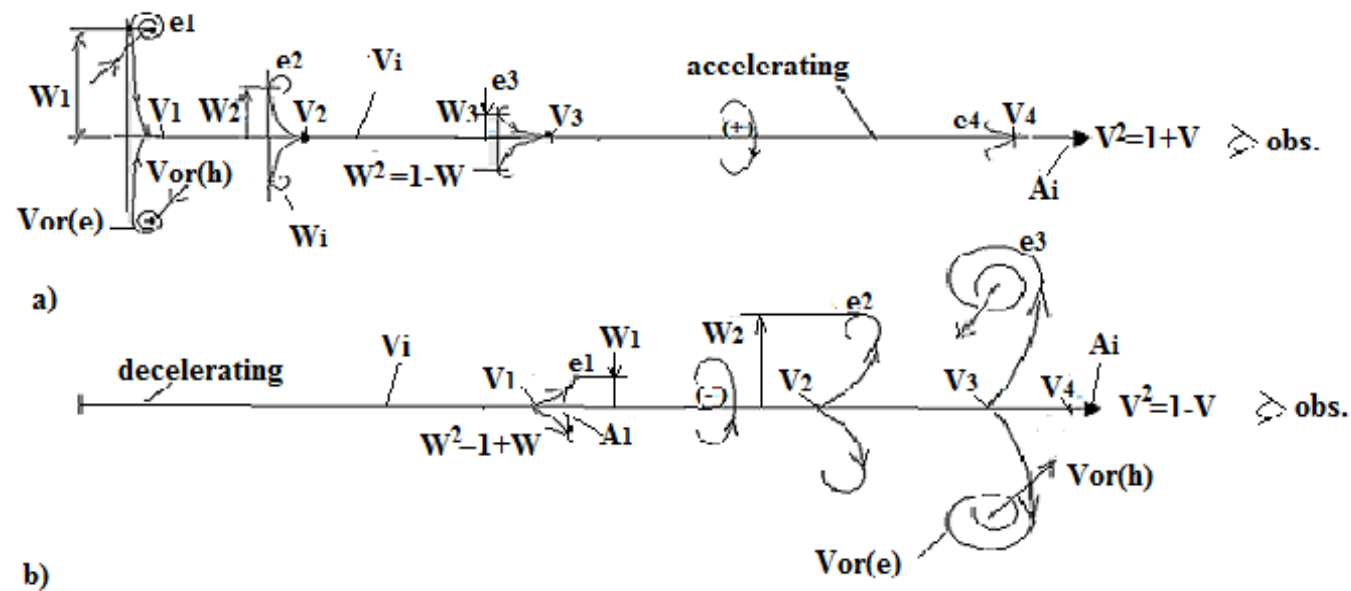

Consequence 1: Accelerating longitudinal vortices wind clockwise $(+)$ and decelerating longitudinal vortices wind counterclockwise (-).

The law $(8 a, b),(9 a, b)$ (point 5.1) shows that when velocity $V_{i}$ increases, the amplitude of cross vortices $\mathrm{W}_{\mathrm{i}}$ decreases (Figure 3a). This phenomenon is due to redistribution of the acceleration of the cross $\left(\mathrm{e}_{\mathrm{i}}\right)$ and longitudinal vortices $\left(\mathrm{A}_{\mathrm{i}}\right)$. Mass is also redistributed: the mass of the cross vortices is added in portions with acceleration to the initial mass of the longitudinal vortex and thus accelerates it.

On the other hand the accelerated longitudinal vortex with acceleration $\left(A_{i}\right)$ sucks in more cross vortices $\left(e_{i}\right)$ from outside that accelerate further the longitudinal vortex $(A)$ and so on. Thus the increase in acceleration and mass at the exit (the longitudinal vortex - $\mathrm{A}_{\mathrm{i}}$ ) translates to an increase in the acceleration and absorption of mass at the entry. This mechanism of amplification is known in cybernetics as Positive Feedback.

Consequence 2: Accelerating longitudinal vortices accelerate and decelerating longitudinal vortices decelerate nonparametrically through Positive Feedback.

When, for example, an accelerating longitudinal vortex sucks in with acceleration a cross vortex, then its first derivative is positive. However the accelerated absorption of the cross vortex slows down and when the positive acceleration of the cross vortex becomes zero, the mass of this cross vortex is added to the longitudinal vortex accelerating it further.

In the next cycle the accelerated longitudinal vortex again sucks in a portion of the cross vortex and so on. Through Positive Feedback the level of saturation constantly increases, the time interval needed for saturation becomes longer, etc. Positive Feedback turns the described avalanche process from an amplifier to a generator.

Consequence 3: The Positive Feedback in a longitudinal vortex turns the process of amplification to a process of generation.

This generative effect of the Positive Feedback was probably used by Nikola Tesla in the construction of the electronic block for his electromobile. The original has worked in generator mode and has needed a battery only at start up. 


\section{Conclusion}

The new axioms offer a new broader basis for building the theory. The expanded theory describes a complete system of uneven dynamic structures. This system contains a chain of transverse (cross) vortices including two mutually orthogonal objects and another chain of longitudinal vortices including two another mutually orthogonal objects. The chain of transverse vortices is perpendicular to the chain of longitudinal vortices. The two chains are connected with two special transformations ( $\Delta 1$ and $\Delta 2)$. Of the two real vortex objects received, one of them describes the Electromagnetic Field. This is the decelerating transverse vortex that emits primary vortices in manifestation of heat. Therefore, Electromagnetic Field is always in the consumer mode.

As one of the vortex objects in the complex system, the role of accelerating vortices is shown for first time. The main feature of accelerating vortices is that they suck in themselves primary vortices. That's why they're always in generation mode. The first of effect is that the accelerating vortex accelerates more and even more .The second effect is that the accelerating vortex attracts, draws or sucks to itself all objects within its range. Extremely strong is the attraction of longitudinal vortices at different speeds which are inserted into each other and form a tube [5] The complex action of these two effects is known in modern physics as Gravity.

\section{References}

[1]. L.D.Landau ,E.M. Lifshitz ,The Classical Theory of Fields ( Volume 2 of A Course of Theoretical Physics ) ,4 Edition., Butterworth-Heinemann,1975.

[2]. V.Markova, The other axioms (Monograph, Book 1, Nautilus, Sofia, 2003)

[3]. V.Markova, three space times obtained by combined vortex movements, International Journal of Current Research, vol.8, iss. 9, p.37826-37832, 2016.

[4]. L.Ting, , Viscous Vortical Flows, ( Lecture notes in physics, Springer-Verlag, 1991)

[5]. V.Markova, A method of movement at a speed greater than the speed of the light, 15-th Conference"Aviation and Cosmonautics", MAI, Moscow, 2016. 8 pages 